\title{
THE VALIDITIES OF PEP AND SOME CHARACTERISTIC FORMULAS IN MODAL LOGIC
}

Hong Zhang, Huacan He

School of Computer Science, Northwestern Polytechnical University,Xi'an, Shaanxi 710032, P.R. China

Abstract: In this paper, we discuss the relationships between some characteristic formulas in normal modal logic with their frames. We find that the validities of the modal formulas are conditional even though some of them are intuitively valid. Finally, we prove the validities of two formulas of PositionExchange-Principle (PEP) proposed by papers 1 to 3 by using of modal logic and Kripke's semantics of possible worlds.

Key words: Characteristic Formulas, Validity, PEP, Frame

\section{INTRODUCTION}

Reasoning about knowledge and belief is an important issue in the research of multi-agent systems, and reasoning, which is set up from the main ideas of situational calculus, about other agents' states and actions is one of the most important directions in $\mathrm{AI}^{[1-3]}$. Papers [1-3] put forward an axiom scheme in reasoning about others(RAO) in multi-agent systems, and a rule called Position-Exchange-Principle (PEP), which is shown as following, was abstracted .

$$
C(\varphi \rightarrow \psi) \rightarrow(C \varphi \rightarrow C \psi) \quad\left(C \in\left\{B_{1}^{S_{1}}, \ldots, B_{m}^{S_{m}}\right\}\right)
$$

When the length of $C$ is at least 2, it reflects the mechanism of an agent reasoning about knowledge of others. For example, if $\mathrm{C}$ is replaced with $B_{j}^{S_{i}} B_{j}^{S_{j}}$, then it should be

$$
B_{i}^{S_{i}} B_{j}^{S_{j}^{j}}(\varphi \rightarrow \psi) \rightarrow\left(B_{i}^{S_{i}} B_{j}^{S_{j}} \varphi \rightarrow B_{i}^{S_{i}} B_{j}^{S_{j}} \psi\right)
$$


The axiom schema plays a useful role as that of modus ponens and axiom $\mathrm{K}$ when simplifying proof. Though examples were demonstrated as proofs in papers [1-3], from the perspectives both in semantics and in syntax, the rule is not so compellent. They had not developed systematic proof for RAO, and paper [1] only assumed that any single modal operator $B_{j}^{s_{j}}$ should satisfy the K45. From the point of intuitive view, the formula $K_{a} p \wedge K_{a}(p \rightarrow q) \rightarrow K_{a} q$ can be proved in epistemic logic systems ${ }^{[5]}$, i.e. the logic subsequence of a epistemic proposition is also known. Thus the logic the omniscience problem is emerged ${ }^{[4-6]}$. From another point of view, because of many different factors such as agents' states both in physical and in mind, the understandings of agents' to PEP are also much different.

Researches in modal logic and the semantics of possible worlds have become a set of theories which are one of the most powerful formal tools in knowledge representing and reasoning or even in intelligent agent and MultiAgent Systems ${ }^{[4]}$. As normal modal systems are formed by adding some axioms on the basis of System $\mathrm{K}$, they are also called expansions of System $\mathrm{K}$. If a new system $\mathrm{S}$ is obtained by adding an axiom, then the axiom is called the characteristic axiom of S, and in sometimes it is also called axiom $\mathrm{S}$ for simplifying.

We analyzed some characteristic axioms (or formulas) in the semantics of possible-worlds, and obtained their corresponding conditions, to which are met, the corresponding axioms are valid. Through analysis and proof, we find that some formulas are conditional valid even through they are valid from an intuitive view. As the validity problems cannot be solved formally in the Modal Logic ${ }^{[7]}$, we prove them in a semantic view.

The rest of the paper is organized as following: Some of the theories, which including the semantics of the possible worlds, the concepts of frame, model and characteristics, etc., are related in Section 2. In Section 3, we prove the validities of some characteristic formulas, such as D,T,B etc., with the corresponding conditions of their frames. In Section 4, we present two corresponding modal formulas to prove the validity of PEP. Finally, we give our conclusions.

\section{RELATED THEORIES}

Modal Logic ${ }^{[7,9]}$ is formed from the classical logic by adding the modal operators, such as $\square$ and $\diamond$ for 'necessity' and 'possibility', as well as the extensions of axioms and rules. Different modal logic calculus systems are formed by choosing different set of axioms. 
Logic

The semantics of the possible-worlds ${ }^{[10]}$ is also called relationship semantics. In the research to necessity or/and possibility of a proposition, we should make clear of the following two important contents:

(1) The proposition to be studied should be in a world, though under the idea of semantics of the possible worlds, its necessity and possibility are depended on the truth-values of other worlds.

(2) The relationships to those possible worlds. Because not all of the possible worlds are 'possible' accessible from the world to be studied, the possible worlds have some relations or conditions with each other. When we study the necessity and/or the possibility of a proposition in a world, we simply concern the "possible" worlds, called accessible worlds, not the unaccessible ones.

There are two opposite views in logic-philosophy school regarding the definitions of possible worlds. While there three famous points of view on how to regard possible worlds and practical ones ${ }^{[8]}$. The theoretical bedrocks of our researches are modal logic and Kripke's semantics of possible worlds, which was established from Leibnitz's theory of possible worlds.

\section{DEFINITION 1: ISSUES RELATED TO THE THEORY OF POSSIBLE WORLDS}

A possible world is one of the possible integration of all of the possible subjects, which are either practical or non-practical worlds. The worlds which are impossible in physics belong to the subset of possible world, while the possible worlds' set in physics are a true subset of all possible worlds' sets, which form a class of possible worlds, denoted by W.

The world an agent stays may be considered as a real world, which is make up of all of the subjects existed. An agent's possible world or state is the integration of its physical states and mental ones.

\section{DEFINITION 2: FRAME AND MODEL}

A frame is a kind of structures which describe the relationships among possible worlds. It can be denoted by a dual $\langle\mathrm{W}, \mathrm{R}\rangle$, where the possible world $\mathrm{W}$ is a non-empty finite set, and $\mathrm{R}$ is a two-place relation $(R \subseteq W \times W)$ on the $\mathrm{W}$. Usually, we call a frame a certain one according to the property of the $\mathrm{R}$. For example, if $\mathrm{R}$ is reflexive, we call the frame a reflexive frame, while a symmetric frame if $\mathrm{R}$ is symmetric,..., and so on. 
A model is a logically rigor explanation to a frame in some specific possible worlds. It is denoted by a dual $\langle\mathrm{F}, \mathrm{V}\rangle$ or a triple $\langle\mathrm{W}, \mathrm{R}, \mathrm{V}\rangle$, where $\mathrm{W}$ and $\mathrm{R}$ are the same to the corresponding frame $\mathrm{F}$, and $\mathrm{V}$ is a dualistic function, which expresses a valuation or assignment of truth values true or false not to a formula but to pairs (w, P) consisting of an element $\mathrm{W}$ of $\mathrm{W}$ and a formula $\mathrm{P}$ in the possible world. Its value-domain is \{true, false $\}$ or $\{0,1\}$.

\section{DEFINITION 3: BASIC SEMANTIC DEFINITION}

Let $\mathrm{M}=<\mathrm{W}, \mathrm{R}, \mathrm{V}>$ be a model, $\mathrm{P}$ and $\mathrm{Q}$ are formulas, then the valuation $V_{M, w}(P)$ on a given $\mathrm{M}, \mathrm{w} \in \mathrm{W}$ is defined as following:

(1) $V_{M, w}(p)=V_{w}(p)$, where $\mathrm{p}$ is a basic meta-proposition;

(2) $V_{M, w}(\neg P)=$ true, if and only if $V_{M, w}(P)=$ fasle;

(3) $V_{M, w}(P \rightarrow Q)=$ true, if and only if $V_{M, w}(P)=$ fasle or $V_{M, w}(Q)=$ true ;

Note that (3) also can be equally expressed as following:

$(3)^{\prime} V_{M, w}(P \rightarrow Q)=$ true, if and only if if $V_{M, w}(P)=$ true, then $V_{M, w}(Q)=$ true

(4) $V_{M, w}(\square P)=$ true, if and only if for every $w^{\prime} \in W\left(w R w^{\prime}\right)$ we have $V_{M, w^{\prime}}(P)=$ true, please see figure 1(a) for reference;

(5) $V_{M, w}(\diamond P)=$ true ,if and only if for some $w^{\prime} \in W\left(w R w^{\prime}\right)$ we have $V_{M, w^{\prime}}(P)=$ true, please see figure $1(\mathrm{~b})$ for reference;

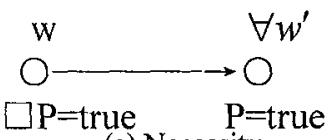

(a) Necessity

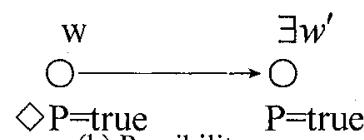

(b) Possibility

figure 1 Necessity and Possibility

Because $\mathrm{P}$ can be a new formula by adding modal operators to the atomic propositions, we have the following deductions:

$(4)^{\prime} \quad V_{M, w}(\square \square P)=$ true, $\quad$ iff for all $w^{\prime} \in W\left(w R w^{\prime}\right)$ we have $V_{M, w^{\prime}}(\square P)=$ true, please see figure 2(a);

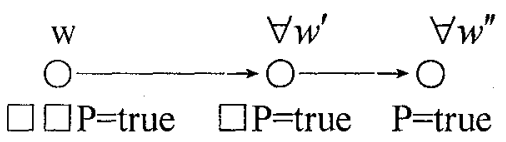

(a) Necessary necessity

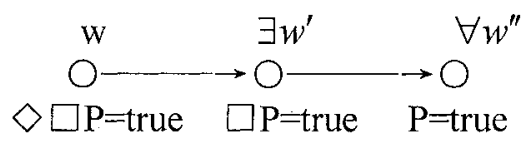

(b) Possible necessity

figure 2 Superposition of modal operators 
Logic

$(5)^{\prime} V_{M, w}(\diamond \square P)=$ true, $\quad$ iff for some $w^{\prime} \in W\left(w R w^{\prime}\right)$ we have $V_{M, w^{\prime}}(\square P)=$ true, please see figure $2(\mathrm{~b})$.

\section{DEFINITION 4: THE SATISFIABILITY AND THE VALIDITY OF A FORMULA}

Let $M=<W, R, V>$ be a model, $w$ a given state in the $\mathrm{W}$ of $\mathrm{M}$. For a arbitrary formula $\mathrm{P}$, if there is a valuation $\mathrm{V}$, such that $V_{M, w}(P)=$ true, then we call that $\mathrm{P}$ is satiable in the $\mathrm{w}$ of the $\mathrm{M}$, denoted by $M \mid={ }_{w} P$. Otherwise, $\mathrm{P}$ is un-satiable in the $\mathrm{w}$ of the $\mathrm{M}$, denoted by $M \mid \neq_{w} P$.

If for all the possible worlds $\mathrm{w}(\mathrm{w} \in \mathrm{W})$ on the $\mathrm{M}$, we always have $V_{M, w}(P)=$ true, then we call that $\mathrm{P}$ is valid on the $\mathrm{M}$, denoted by $M \mid=P$. Otherwise, $\mathrm{P}$ is un-valid on the $\mathrm{M}$, denoted by $M \mid \neq P$.

Furthermore, $\mathrm{P}$ is valid on a frame $\mathrm{F}$ if $\mathrm{P}$ is valid on a class of model based on $\mathrm{F}$.

\section{DEFINITION 5: THE PROPERTIES OF A FRAME}

A frame (or model) usually has many properties, which can be strictly expressed by a formal way. For example, if a formula $\mathrm{P}$ is true under an arbitrary valuation $\mathrm{V}$ in all of the $\mathrm{w}(\mathrm{w} \in \mathrm{W})$, i.e. $\mathrm{P}$ is valid on $\mathrm{F}$, then for every $\mathrm{W}$, it must has a accessible world $w^{\prime}$. This case can be denoted by: $(\forall w)\left(\exists w^{\prime}\right)\left(w R w^{\prime}\right)$.

A relation which meets such kind of condition is called a serial one, and a frame with a $\mathrm{R}$ of serial relation is called a serial frame.

Four of other most frequently used properties of a frame are formally given as followings:

$\begin{array}{ll}\text { reflexivity } & (\forall w)(w R w) \\ \text { symmetry } & (\forall w)\left(\forall w^{\prime}\right)\left(w R w^{\prime} \rightarrow w^{\prime} R w\right) \\ \text { transitivity } & (\forall w)\left(\forall w^{\prime}\right)\left(\forall w^{\prime \prime}\right)\left(w R w^{\prime} \wedge w^{\prime} R w^{\prime \prime} \rightarrow w R w^{\prime \prime}\right) \\ \text { Euclidean } & (\forall w)\left(\forall w^{\prime}\right)\left(\forall w^{\prime \prime}\right)\left(w R w^{\prime} \wedge w R w^{\prime \prime} \rightarrow w^{\prime} R w^{\prime \prime}\right)\end{array}$

\section{CHARACTERISTIC FORMULAS:}

Formal systems of modal logic are established according to either a formula's validity or its non-contrariness. They are divided into normal systems and non-normal systems according to whether they contain axiom $\mathrm{K}$ and necessitation (another common name for it is generalization) rule. The 
followings are 5 of the most important characteristic formulas (or axioms) of modal logic.

$\mathrm{D}: \square \mathrm{p} \rightarrow \diamond \mathrm{p}$

T: $\square \mathrm{p} \rightarrow \mathrm{p}$

B: $p \rightarrow \square \diamond p$

A: $\square \mathrm{p} \rightarrow \square \square \mathrm{p}$

$\mathrm{E}: \diamond \mathrm{p} \rightarrow \square \diamond \mathrm{p}$

Where, $D$ and $T$ are intuitively valid formulas. The mean of $D$ is that if $p$ is necessary, then it is possible. In some degree, D reflects the law of "The ought should be approved" in deontic logic. The mean of $T$ is that if $p$ is necessarily true, then $\mathrm{p}$ is true. Or in a nutshell, the necessary is the practical. $T$ is called necessitation axiom as well.

$\mathrm{B}, \mathrm{A}$ and $\mathrm{E}$ are the formulas which cannot be ascertained its validity on intuitively. The mean of $B$ is that if $p$ is true, then $p$ is necessarily possible. The mean of $A$ is that if $p$ is necessary, then the necessity of $p$ is necessary. The mean of $\mathrm{E}$ is that if $\mathrm{p}$ is possible, then the necessity of $\mathrm{p}$ is possible. Though these points of view are sounded, their validities are not so evident.

There are also some intuitively in-valid formulas, such as $\mathrm{p} \rightarrow \square \mathrm{p}, \diamond$ $p \rightarrow p$, etc. which are also belong to modal logic formulas, because they can form theoretically meaningful systems according to non-contrariness rule.

If a characteristic axiom is to be valid on a frame, the $\mathrm{R}$ of the frame must meet a certain condition. We often ignore the corresponding conditions of some formulas which are not intuitively valid because of the intuitively validity of $\mathrm{D}$ and $\mathrm{T}$. The followings are some of the proofs we give to the characteristic axioms on their frames.

\section{THE VALIDITIES OF SOME CHARACTERISTIC FORMULAS ON THEIR FRAMES}

We will discuss the relationships between the validities of the 5 characteristic formulas (or axioms) with the corresponding properties of their frames (or models), respectively.

\section{PROPOSITION 1: AXIOM D IS SOUND AND COMPLETE FOR SERIAL MODELS}

Proof. Let's prove the soundness first, i.e. $D(\square p \rightarrow \diamond p)$ is sound for serial models. Because for every valuation $\mathrm{V}$ on a serial frame and to every possible world $\mathrm{w}$, if $V_{M, w}(\square p)=$ true, as $\mathrm{R}$ is serial, there will be $w, w R w^{\prime}$, such that $V_{M, w^{\prime}}(p)=$ true. According to the definition of possibility 
Logic

(Definition 3(5)), we get that when p is true on the accessible world $w^{\prime}, \diamond \mathrm{p}$ is true on $w$. therefore $V_{M, w}(\square p \rightarrow \diamond p)=$ true. Since $V$ and $w$ are both arbitrary, $\mathrm{D}$ is valid on the frame.

Now let's prove the completeness, i.e. $D(\square p \rightarrow \diamond p)$ is complete for serial models. Let $\mathrm{F}=<\mathrm{W}, \mathrm{R}>$ be an arbitrarily given serial frame. For an arbitrary $\mathrm{w}$ from set $\mathrm{w} \in \mathrm{W}$, if $V_{M, w}(\square p \rightarrow \diamond p)=$ true, then according to definition 3(3), we get

$$
\begin{aligned}
V_{M, w}(\square p) & =\text { false } \\
\text { or } \quad V_{M, w}(\diamond p) & =\text { true }
\end{aligned}
$$

We apply definition 3(4) to (1), there exists a $w, w R w^{\prime}$, and

$$
V_{M, w^{\prime}}(p)=\text { false }
$$

and we apply definition 3(5) to (2), there exists a $w^{\prime \prime}, w R w^{\prime \prime}$, and

$$
V_{M, w^{\prime \prime}}(p)=\text { true }
$$

We can see from expressions (3) and (4) that if we wish $w$ be true in $\mathrm{w}$, there must be an accessible world $w^{*}$, regardless either it is $w^{\prime}$ or $w^{\prime \prime}$. Because whether $w^{*}$ is any of them, the proposition that $\mathrm{p}$ is true or false is always true in $w^{*}$. i.e. (3) or (4) is always valid. According to the serial property of a frame, if $\mathrm{D}$ is desired to be valid on a frame, then the frame must be serial.

\section{PROPOSITION 2: AXIOM T IS SOUND AND COMPLETE FOR REFLEXIVE MODELS}

Proof. Let's prove the soundness first, i.e. $\mathrm{T}(\square p \rightarrow p)$ is sound for reflexive models. Let $\mathrm{M}$ be a model with a reflexive relation. If in a possible world $w(w \in W)$, we have $V_{M, w}(\square p)=$ true, then for all $w^{\prime}\left(w R w^{\prime}\right)$, we get $V_{M, w^{\prime}}(p)=$ true. As model $\mathrm{M}$ is reflexive, i.e. $(\forall w)(w R w)$, we have $V_{M, w}(p)=$ true. Therefore we get $M \mid=_{w} \square p \rightarrow p$. For $w$ is arbitrary, we get $M \mid=\square p \rightarrow p$. Furthermore, $\square p \rightarrow p$ is valid in a frame with a reflexive relation.

Then we come to the completeness, i.e. T $(\square p \rightarrow p)$ is complete for reflexive models. Let's construct a counter-example of $M \mid=\square p \rightarrow p$, which implies the frame is not reflexive. Let $M$ be a model without the property of reflexive, i.e. there is a $w(w \in W)$ in which $(w R w)$ is invalid. In other words, assume that $\mathrm{F}$ is a non-reflexive frame, then there must be a state $w(w \in W)$, such that $(w R w)$ does not hold.

We will try to falsify $\square p \rightarrow p$ in $w$. For this purpose we have to find a valuation $\mathrm{V}$ such that $V_{M, w}(\square p)=$ true but $V_{M, w}(p)=$ false, thus we get $V_{M, w}(\square p \rightarrow p)=$ false. i.e. we have indeed found a valuation $V$ and a state 
$\mathrm{w}$ such that $\square p \rightarrow p$ does not hold in $\mathrm{w}$. Therefore $\mathrm{T}$ is not valid in $\mathrm{F}$. This proves the completeness.

\section{PROPOSITION 3: AXIOM B IS SOUND AND COMPLETE FOR SYMMETRIC MODELS}

Proof. We prove the soundness first, i.e. B $(p \rightarrow \square \triangleright P)$ is sound for symmetric models. If $M \mid=_{w} p$, then for any $w^{\prime}\left(w R w^{\prime}\right)$ we have $M \mid=_{w^{\prime}} \bigcirc p$, and $M \mid=_{w} \square\left\langle p\right.$ by symmetry $(\forall w)\left(\forall w^{\prime}\right)\left(w R w^{\prime} \rightarrow w^{\prime} R w\right)$. Thus we get $M \mid=_{w} p \rightarrow \square \bigcirc p$. Furthermore, as $w$ is arbitrary, we get $M \mid=p \rightarrow \square \backslash P$.

Then let's come to the completeness, i.e. B $(p \rightarrow[K>P)$ is complete for symmetric models. Assume $V_{M, w}(p \rightarrow \square \triangleright p)=$ true, i.e. if $V_{M, w}(p)=$ true then $V_{M, w}(\square \otimes p)=$ true. The latter can be also interpreted as: for all $w^{\prime}$, if $w R w^{\prime}$ then $V_{M, w^{\prime}}(\diamond p)=$ true. Applying the premise, we obtain if $V_{M, w}(p)=$ true then $V_{M, w^{\prime}}(\diamond p)=$ true .From the definition of possibility, we can see that $w$ is accessible to $w^{\prime}$, i.e. $w^{\prime} R w$. Therefore we obtain $(\forall w)\left(\forall w^{\prime}\right)\left(w R w^{\prime} \rightarrow w^{\prime} R w\right)$.

\section{PROPOSITION 4: AXIOM A IS SOUND AND COMPLETE FOR TRANSITIVE MODELS}

Proof. We prove the soundness first, i.e. A $(\square \mathrm{p} \rightarrow \square \square \mathrm{p})$ is sound for transitive models. If $M \mid=_{w} \square p$, then for any $w^{\prime}\left(w R w^{\prime}\right), M \mid=_{w^{\prime}} p$. And it is the case that for any $w^{\prime \prime}\left(w^{\prime} R w^{\prime \prime}\right)$ we have $w R w^{\prime \prime}$ by transitivity, and so $M \mid=_{w^{\prime \prime}} p$. Thus $M \mid=_{w^{\prime}} \square p$ for any $w^{\prime}\left(w R w^{\prime}\right)$, and $M \mid=_{w} \square \square p$. Thus $M \mid={ }_{w} \square p \rightarrow \square \square p$. Further, for $\mathrm{w}$ is arbitrarily given, we get $M \mid=\square p \rightarrow \square \square p$.

Then we come to the completeness, i.e. A ( $\square \mathrm{p} \rightarrow \square \square$ p) is complete for transitive models. Let $w R w^{\prime}$ if and only if whenever a formula $\square \mathrm{p}$ is in $w$, the formulas $\square \mathrm{p}$ and $\mathrm{p}$ are both in $w^{\prime}$. Then $\mathrm{R}$ will be transitive. For if $(\forall w)\left(\forall w^{\prime}\right)\left(\forall w^{\prime \prime}\right)\left(w R w^{\prime} \wedge w^{\prime} R w^{\prime \prime} \rightarrow w R w^{\prime \prime}\right)$, then whenever $\square \mathrm{p}$ is in $\mathrm{w}$, $\square \mathrm{p}$ and $\mathrm{p}$ will be in $w^{\prime}$, and since the former is in $w^{\prime}$ both will also be in $w^{\prime \prime}$, so we get $w R w^{\prime \prime}$.

\section{PROPOSITION 5: AXIOM E IS SOUND AND COMPLETE FOR EUCLIDEAN MODELS}

The reasoning procedures are same to the above, omitted. 
Logic

\section{THE VALIDITY OF PEP}

Paper [1-3] presented a reasoning pattern regarding reason about knowledge of others in multi-agent systems ---- Position-Exchange-Principle, which is formalized as the following axiom schema:

$$
C(\varphi \rightarrow \psi) \rightarrow(C \varphi \rightarrow C \psi)
$$

Where $C \in\left\{B_{1}^{S_{1}}, \ldots, B_{m}^{S_{m}}\right\}$, and the length of $C$ is at least 2. When $C=\varepsilon,(5)$ is a variant of the so-called modus ponens in propositional logic and predicate logic. When $C=B_{i}^{S_{i}}$, (5) is just the axiom (K) in knowledge logic. When the length of $\mathrm{C}$ is at least 2, (5) reflects the mechanism of one agent reasoning about knowledge of another agent. For example, if $C=B_{i}^{S_{i}} B^{S_{j}}$, then (5) should be

$$
B_{i}^{S_{i}} B_{j}^{S_{j}}(\varphi \rightarrow \psi) \rightarrow\left(B_{i}^{S_{i}} B_{j}^{S_{j}} \varphi \rightarrow B_{i}^{S_{i}} B_{j}^{S_{i}} \psi\right)
$$

Because equivalent propositions are not equivalent as belief ${ }^{[1]}$, PEP, which makes the reasoning procedures of reasoning about others in MAS simple and clear, may probably imply a character which is counter-intuitive. Though paper[1-3] demonstrated examples to support their theories, we think it is not sufficed. From our analyzing in Section 3, many formulas are conditional valid even if they are valid on intuitions.

As the necessitation operator $\square$ represents an agent's belief in the general BDI model ${ }^{[12]}$, in the following, we will prove the validity of two basic modal formulas which support PEP.

\section{PROPOSITION 6: FORMULA $\square(p \rightarrow q) \rightarrow(\square p \rightarrow \square q)$ IS VALID FOR THE CLASS OF ALL MODELS}

Proof. Let $\mathrm{M}$ be any model, $\mathrm{w}$ an arbitrarily given state in $\mathrm{W}$. If $M \mid \neq_{w} \square(p \rightarrow q) \quad, \quad$ then $M \mid={ }_{w} \square(p \rightarrow q) \rightarrow(\square p \rightarrow \square q)$. If $M \mid=_{w} \square(p \rightarrow q)$ and $M \mid \neq_{w} \square p$, then $M \mid=_{w}(\square p \rightarrow \square q)$, Hence $M \mid={ }_{w} \square(p \rightarrow q) \rightarrow(\square p \rightarrow[\square q)$. So we need only to prove $M\left|=_{w} \square(p \rightarrow q) \& M\right|=_{w} \square p \rightarrow M \mid=_{w} \square q$.

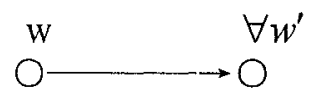
(a) The necessity of an implication

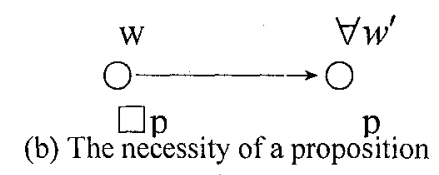

Figure 3 The necessities of formulas

If $M \mid=_{w} \square(p \rightarrow q)$ and $M \mid=_{w} \square p$, then for any $w^{\prime}\left(w R w^{\prime}\right) \quad$ we have $M \mid=_{w^{\prime}}(p \rightarrow q) \quad$ (please see figure 3(a)) and $M \mid=_{w^{\prime}} p$ (please see figure 3(b)). Hence $M \mid=_{w^{\prime}} q$ for any $w^{\prime}\left(w R w^{\prime}\right)$, and $M \mid=_{w} \square q$. So $M \mid=_{w} \square(p \rightarrow q) \rightarrow(\square p \rightarrow \square q)$. 
Since $\mathrm{M}$ is any model, and $\mathrm{w}$ an arbitrarily given state in $\mathrm{W}$, $\square(p \rightarrow q) \rightarrow(\square p \rightarrow \square q)$ is valid for the class of all frames.

\section{PROPOSITION7: FORMULA $\square \square(p \rightarrow q) \rightarrow(\square \square p \rightarrow \square \square q)$ IS VALID FOR THE CLASS OF ALL MODELS}

Proof. Similar to the above, we only prove $M\left|=_{w} \square \square(p \rightarrow q) \& M\right|=_{w} \square \square p \rightarrow M \mid=_{w} \square \square q$.

For an arbitrarily given state $w^{\prime}\left(w R w^{\prime}\right)$, if $M \mid=_{w} \square \square(p \rightarrow q)$, we get $M \mid=_{w^{\prime}} \square(p \rightarrow q)$; and for another arbitrarily given state $w^{\prime \prime}\left(w^{\prime} R w^{\prime \prime}\right)$, we also have $M \mid==_{w^{\prime \prime}}(p \rightarrow q)$ (please see figure 4(a) for reference). And by the some procedure, we have $M \mid=_{w^{\prime}} \square p$ and $M \mid=_{w^{\prime \prime}} p$ from $M \mid=_{w^{\prime \prime}} \square \square p$ (please see figure 4(a) for reference). Hence $M \mid=_{w^{\prime \prime}} q$. Since $w^{\prime \prime}$ and $w^{\prime}$ are both arbitrary, we get $M \mid=_{w^{\prime}} \square q$; and as $w^{\prime}$ and w are both arbitrary possible worlds or states, we obtain $M \mid=_{w} \square \square q$. Thus $M \mid=_{w} \square \square(p \rightarrow q) \rightarrow(\square \square p \rightarrow \square \square q)$.Because $\mathrm{M}$ and $\mathrm{w}$ are both arbitrarily chosen, $\square \square(p \rightarrow q) \rightarrow(\square \square p \rightarrow \square \square q)$ is valid for the class of all frames.

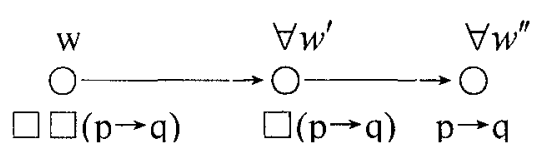

(a) The necessary necessity of an implication

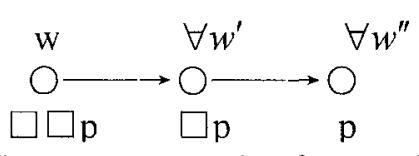

(b) The necessary necessity of a proposition

Figure 4 The necessary necessities of formulas

\section{CONCLUSIONS}

By analyzing the characteristic axioms using the possible-world semantics, we obtained some of their corresponding conditions, saying corresponding formulas. These formulas can be regarded as the formal explanations of the characteristic axioms. On the other hand, only when these conditions are met, are valid the corresponding formulas (or axioms). In this way, we can make it sure that which axioms are valid for the right thinking formulas at a specific condition. Through analyzing, we discover that some formulas, such as $\mathrm{D}$ and $\mathrm{T}$, are conditional valid even through they are valid on intuition. As the necessitation operator $\square$ represents an agent's belief in the general BDI model, we analyzed the validity of PEP proposed by papers [1-3], and proved the validity of PEP from the points of modal logic and Kripke's semantics of possible worlds. 
Logic

PEP is very useful in multi-agent epistemic logic. It plays an important role in the knowledge research of MAS as that of modus ponens and axiom $\mathrm{K}$ do in propositional logic and knowledge logic. Though we have proved the validity of PEP on any frames, it is a reasonable reasoning of an agent reasoning about knowledge of others in the default of the premise that she knows the states and actions of herself and the agent about whom she reasons. If an agent doesn't know her states or actions (i.e. epistemic reflexive axioms are not met), or the epistemic states of the two agents are much different, then PEP is probably not so valid. Therefore, in practical, when we use PEP as a good tool to reason about others, it is wise to make sure or clear that any single modal operator satisfies the KD45.

\section{REFERENCES}

[1] SHI Zhongzhi,TIAN Qijia and LI Yunfeng. RAO Logic for Multi-agent Framework. Journal of Computer Science and Technology, 1999,14(4):393-400

[2] WANG Wenjie,TIAN Qijia and SHI Zhongzhi.Reasoning About Others in MAS(In Chinese).Journal of Computer Research and Development.1998,35(11):971-974

[3] TIAN Qijia, SHI Zhongzhi and WANG Huaiqing. Reasoning About Actions and Knowledge in MAS(In Chinese). In: Proceeding of The Fourth Chinese Joint conference on Artificial Intelligence.Beijing:Tsinghua University Press, 1996:74-79

[4] YANG Kun,CHEN Jianzhong,SUN Degang,LIU Dayou. Logic Omniscience Problem in Epistemic Logics and the Methods to Resolve It. Journal of Jilin University(Natural Sciences), 1999(3):40-43

[5] ZHOU Changle. A Introduction to Epistemic Logics(In Chinese). Beijing:Tsinghua University Press, 2001

[6] LI Jinhou, JIANG Jinping. Two Blind Spots in Recent Logic-Based Agent Study Inferred from the Declaration of Logic Omniscience Problem(In Chinese). Journal of University of Science and Technology Beijing, 2004,26(2):215-218

[7] ZHOU Beihai. Modal Logics(In Chinese). Beijing: China Social Science Press, 1997

[8] HU Zehong. The Philosophical reflectons of Logics(In Chinese). Beijing: Central Editorial Press, 2004:127-135

[9] Patrick Blackburn, Maarten de Rijke, Yde Venema. Modal Logic. Cambridge University Press, 2001

[10] S.Kripke. Semantic Analysis of Modal Logic II : Non-Normal Modal Propositional Calculi. In:Symposium on the Theory of Models,North-Holland,Amsterdam, 1965

[11] Konolige,K. A Deduction Model of Belief. Pitman,London and Morgan Kaufmann,San Mateo,CA. 1986

[12] LIU Yong,PU Shuzhen,CHENG Daijie,and CAO Zehan.Belief Characteristic's of BDI Model(In Chinese).Journal of Computer Research and Development.2005,42(1):54-59 